\title{
A type $B$ analog of the Lie representation
}

\author{
Andrew Berget $\left.\right|^{\dagger}$
}

Department of Mathematics, Western Washington Univeristy, Bellingham, Washington, USA

\begin{abstract}
We describe a type B analog of the much studied Lie representation of the symmetric group. The $n$th Lie representation of $S_{n}$ restricts to the regular representation of $S_{n-1}$, and our generalization mimics this property. Specifically, we construct a representation of the type B Weyl group $B_{n}$ that restricts to the regular representation of $B_{n-1}$. We view both of these representations as coming from the internal zonotopal algebra of the Gale dual of the corresponding reflection arrangements.

Résumé. Nous décrivons un type B analogue de la bien connue représentation Lie du group symmetrique. Le représentation Lie est un représentation de $S_{n}$ qui restreint à la représentation régulière de $S_{n-1}$, et notre géneralisation imite cette propriété. Plus précisément, nous construisons une représentation de group Weyl $B_{n}$ qui restreint à la représentation régulière de $B_{n-1}$. Ces deux représentations sont des occurrences de l'algèbre interne zonotopal de dual Gale des arrangements de réflexion respectifs.
\end{abstract}

Keywords. decreasing trees, hyperplane arrangements, Lie representation, hyperoctohedral group, zonotopal algebra

\section{Introduction}

We consider the polynomial ring $S$ in two sets of variables $y_{i j}$ and $z_{i j}$, where $1 \leq i<j \leq n$. We have the following conventions on the indices: For all $1 \leq i, j \leq n$ with $i \neq j, y_{j i}=-y_{i j}$ and $z_{j i}=z_{i j}$.

This ring has an action of the Weyl group of type $\mathrm{B}$, which we denote by $B_{n}$. Recall that this is the group of signed permutations of $n$ letters, which are permutations $w$ of $\{1, \overline{1}, 2, \overline{2}, \ldots, n, \bar{n}\}$ satisfying the rule $w(\bar{i})=\overline{w(i)}$ for all $1 \leq i \leq n$. The action of $(i j)(\overline{i j})$ on $S$ is by permuting the indices of the $y$ 's and $z$ 's, while the action of $(k \overline{\bar{k}})$ is by the rules

$$
(k \bar{k}) y_{i j}=\left\{\begin{array}{ll}
y_{i j} & k \notin\{i, j\}, \\
z_{i j} & k=i, \\
-z_{i j} & k=j,
\end{array} \quad(k \bar{k}) z_{i j}= \begin{cases}z_{i j} & k \notin\{i, j\}, \\
y_{i j} & k=i, \\
-y_{i j} & k=j .\end{cases}\right.
$$

The main result of our work is the following.

Theorem 1.1 Define an ideal in $S=\mathbf{C}\left[y_{i j}, z_{i j}: 1 \leq i<j \leq n\right]$ by

$$
\begin{array}{r}
J=\left\langle y_{i j}^{2}, z_{i j}^{2}, y_{i j} z_{i j}: 1 \leq i<j \leq n\right\rangle+\left\langle y_{j i} y_{i k}+y_{i j} y_{j k}+y_{i k} y_{k j},\right. \\
\left.\quad z_{j i} y_{i k}-z_{i j} z_{j k}-y_{i k} z_{k j}: 1 \leq i<j<k \leq n\right\rangle
\end{array}
$$

\footnotetext{
${ }^{\dagger}$ Email: andrew. berget @wwu.edu

1365-8050 @ 2016 Discrete Mathematics and Theoretical Computer Science (DMTCS), Nancy, France
} 
Then, $J$ is $B_{n}$ stable. The quotient $S / J$ has Hilbert series

$$
(1+2 q)(1+4 q) \cdots(1+(2 n-2) q) .
$$

The top degree component of $S / J$ restricts to the regular representation of $B_{n-1}$.

We propose the top degree component of the quotient $S / J$ as a type B analog of the Lie representation of the symmetric group $S_{n}, \mathrm{Lie}_{n}$. One definition of $\mathrm{Lie}_{n}$ is as the multilinear component of the free Lie algebra on $n$ generators. $\mathrm{Lie}_{n}$ arises in the study of the cohomology of configuration spaces as well as in poset homology. As we will see, there is a general construction for hyperplane arrangements that yields both the Lie representation, and the quotient in Theorem 1.1

Remark 1.2 There is a well-studied representation of $B_{n}$ that bears a striking similarity to the one in Theorem 1.1. the Orlik-Solomon algebra of the reflection arrangement of type B/C. This is a model for the cohomology of the complexified complement of this arrangement $[O S]$, and is different than the construction in Theorem 1.1 in a few fundamental ways.

Most naively, the Orlik-Solomon algebra is a skew-commutative algebra on $n^{2}$ generators. The quotient in Theorem 1.1 is honestly commutative on $n(n-1)$ generators. The primal object for the Orlik-Solomon algebra is the type B arrangement itself, while for Theorem 1.1 it will turn out to be the Gale dual of this arrangement.

The Hilbert series of the Orlik-Solomon algebra of the type B reflection arrangement is

$$
(1+q)(1+3 q) \cdots(1+(2 n-1) q)
$$

and its top degree component has dimension $1 \cdot 3 \cdot 5 \cdots(2 n-1)$. This is the dimension of the whole quotient occurring in Theorem 1.1] not a single graded piece. There is no obvious gradation of the top cohomology of the Orlik-Solomon algebra.

The representation-theoretic properties of the Orlik-Solomon algebra of a reflection arrangement of a root system were studied by Lehrer and Solomon [LS] and in type B specifically by Douglas [Do]. Work of Bergeron [B]] connects a hyperoctohedral analog of the Lie representation to the Orlik-Solomon algebra of the hyperoctohedral arrangement. He proves an isomorphism between this generalized Lie representation and a twisted Orlik-Solomon algebra.

There is a $B_{n}$ invariant submodule of the Orlik-Solomon algebra of the reflection arrangement of $B_{n}$ whose restriction to $B_{n-1}$ is the regular representation. This is the natural candidate to relate the top degree component of $S / J$ to. Already for $n=4$ one finds that the characters of these $B_{n}$ representations differ. Indeed the character of $S / J$ evaluated at $(12)(34)(\overline{1} \overline{2})(\overline{3} \overline{4})$ is 0 while the character of the potential analog in the Orlik-Solomon algebra is -8 . The character of $S / J$ will be computed in future work.

The quotient ring of Theorem 1.1 is the so-called internal zonotopal algebra of the type B reflection arrangement. These algebras were studied by Holtz and Ron [HR], where connections to box splines are made, and later by Ardila and Postnikov [AP10, AP15], among others.

The structure of the paper is as follows. We first review background on zonotopal algebra and Gale duality. Next, we motivate Theorem 1.1 by studying the internal zonotopal algebra of the Gale dual of the braid arrangement. This makes a connection to the well-studied Whitehouse representation of the symmetric group through work of Mathieu [Ma], Gaiffi [Ga], and Robinson and Whitehouse [RW]. We then study the internal zonotopal algebra of the Gale dual of the type B reflection arrangement and prove Theorem 1.1. Lastly, we propose the study of decreasing \pm trees, which are a type B analog of decreasing trees. 


\section{Background}

First we review results of Ardila-Postnikov and Holtz-Ron on so-called zonotopal algebra, which we will later specialize. Following this we recall the notion of Gale duality.

\subsection{Zonotopal algebras of hyperplane arrangements}

Let $\mathcal{A}$ be a central arrangement of hyperplanes in a complex finite dimensional vector space $V$. We let $M=M(\mathcal{A})$ denote the matroid of $\mathcal{A}$. Recall that this is the matroid of the linear forms defining the arrangement, where a set of linear forms is independent when it is a linearly independent set of vectors.

Define a function $\rho_{\mathcal{A}}: V \rightarrow \mathbf{N}$ by the rule

$$
\rho_{\mathcal{A}}(h)=\text { the number of hyperplanes in } \mathcal{A} \text { not containing } h,
$$

and use this to define the ideal

$$
I_{\mathcal{A}, k}=\left\langle h^{\rho_{\mathcal{A}}(h)+k+1}: h \in V\right\rangle \subset \operatorname{Sym}(V) .
$$

For $k \geq 2$ the quotient $\operatorname{Sym}(V) / I_{\mathcal{A}, k}$ has Krull dimension zero, and so it is a finite dimensional complex vector space. The vector space dimension of this quotient is also called the degree. The ring $\operatorname{Sym}(V)$ is graded in the usual way, and we denote the $m$ th graded piece of a graded module over this ring by $(-)_{m}$. The ideal $I_{\mathcal{A}, k}$ is graded, since it is generated by powers of linear forms, which are homogeneous. The Hilbert series of $\operatorname{Sym}(V) / I_{\mathcal{A}, k}$ is the generating function for the dimensions of the graded pieces of this graded $\operatorname{Sym}(V)$-module.

We will describe the Hilbert series of $\operatorname{Sym}(V) / I_{\mathcal{A}, k}$ in terms of the matroid of $\mathcal{A}$. To do so, we need the Tutte polynomial of a matroid. We take the most expedient route: The Tutte polynomial of a matroid $M$ with ground set $E$ and rank function rk $: 2^{E} \rightarrow \mathbf{N}$ is the bivariate polynomial

$$
T_{M}(x, y)=\sum_{A \subset E}(x-1)^{\mathrm{rk}(E)-\mathrm{rk}(A)}(y-1)^{|A|-\mathrm{rk}(A)} .
$$

Theorem 2.1 (Ardila-Postnikov [AP10], Holtz-Ron [HR]) Let $M=M(\mathcal{A})$ denote the matroid of the arrangement $\mathcal{A}$, which consists of $m$ hyperplanes. The Hilbert series of the quotient $\operatorname{Sym}(V) / I_{\mathcal{A}, k}$ is equal to

1. $q^{m-\mathrm{rk}(M)} T_{M}(1+q, 1 / q)$ if $k=0$;

2. $q^{m-\operatorname{rk}(M)} T_{M}(1,1 / q)$ if $k=-1$;

3. $q^{m-\operatorname{rk}(M)} T_{M}(0,1 / q)$ if $k=-2$.

The quotients $\operatorname{Sym}(V) / I_{\mathcal{A}, k}$ occurring in the theorem are, respectively, referred to as the external, central and internal zonotopal algebras of $\mathcal{A}$. The most subtle case of the theorem is the third [AP15]. In the first two cases explicit bases of the Macaulay inverse system of $I_{\mathcal{A}, k}$ can be given [AP10], while in the case $k=-2$ there is no known canonical basis described by the matroid $M$. Naturally, the third case is the one that interests us in the sequel. 


\subsection{Gale duality}

Definition 2.2 Given a r-by-m matrix $N$, let $N^{\perp}$ be any matrix whose rows span the (right) kernel of $N$. We call $N^{\perp}$ a Gale dual of $N$. If $\mathcal{A}$ is an arrangement in $\mathbf{C}^{r}$ whose defining linear forms are the columns of $N$, then $\mathcal{A}^{\perp}$ is an arrangement whose defining linear forms are the columns of some $N^{\perp}$.

There is considerable flexibility in determining the Gale dual of a matrix. If we insist that $N^{\perp}$ be a full rank matrix then it is uniquely determined modulo the left action of the general linear group by matrix multiplication. Note that $N^{\perp}$ will have dimensions $(m-\operatorname{rank}(N))$-by-m, and that $N$ is always a Gale dual of $N^{\perp}$. If there is a block-matrix decomposition $N=[I \mid A]$ then $N^{\perp}=\left[-A^{t} \mid I\right]$ will be Gale dual of $N$.

Gale duality is a geometric realization of matroid duality, which we now explain. If $M$ is a matroid with ground set $E$ then one can construct a new matroid $M^{\perp}$, the dual matroid, whose bases (i.e., maximal independent sets) are complements of bases of $M$. This is an honest duality in the sense that $\left(M^{\perp}\right)^{\perp}=$ $M$. One has $M\left(\mathcal{A}^{\perp}\right)=M(\mathcal{A})^{\perp}$.

The following relation holds between the Tutte polynomials of $M$ and $M^{\perp}$ :

$$
T_{M^{\perp}}(x, y)=T_{M}(y, x) .
$$

It follows that the Hilbert series of $\operatorname{Sym}(V) / I_{\mathcal{A}^{\perp},-2}$ is given by $q^{\mathrm{rk}(\mathcal{A})} T_{M(\mathcal{A})}(1 / q, 0)$.

\section{The braid arrangement}

In this section we introduce the braid arrangement. We apply the construction of Section 2.1 to its Gale dual, which we do to motivate the results that will follow.

\subsection{The braid arrangement}

The braid arrangement in $\mathbf{C}^{n+1}$ is the arrangement $\mathcal{A}_{n+1}$ defined by the linear forms

$$
x_{j}-x_{i}, \quad(1 \leq i<j \leq n+1) .
$$

It is the reflection arrangement of the type A Weyl group, $S_{n+1}$. The natural action of the symmetric group $S_{n+1}$ on $\mathbf{C}^{n+1}$ passes to $\mathbf{C}\left[x_{1}, \ldots, x_{n+1}\right]$, and the ideals $I_{\mathcal{A}_{n+1}, k}$ are invariant under this action. The Macaulay inverse system of $I_{\mathcal{A}_{n+1},-1}$ has dimension $n^{n-2}$, and was studied by the author and Rhoades in [BR]. There it was shown to be a representation of the symmetric group $S_{n+1}$ that restricted to the well studied parking representation of $S_{n}$. This is the representation with basis give by sequences $\mathbf{p}=$ $\left(p_{1}, \ldots, p_{n}\right)$ whose non-decreasing rearrangement $\mathbf{q}$ satisfies $q_{j} \leq j$ for all $1 \leq j \leq n$.

One can choose the Gale dual of $\mathcal{A}_{n+1}$ so that it too has a natural $S_{n+1}$ action. This is most easily described in terms of graph theory. The arrangement $\mathcal{A}_{n+1}$ is the graphic arrangement of the complete graph on $n+1$ vertices, $K_{n+1}$. The matrix of normal vectors of the hyperplanes in $\mathcal{A}_{n+1}$ is the incidence matrix of $K_{n+1}$. The (right) kernel of this matrix is well known to be the cycle space of $K_{n+1}$. This is the subspace of the edge space spanned by oriented cycles in the graph.

Recall that the edge space of $K_{n+1}$ is the complex vector space with distinguished basis $e_{i j},(1 \leq i<$ $j \leq n+1)$. We identify the edge space with its dual, defining $\left\{e_{i j}\right\}$ to be an orthonormal basis. We set $e_{i j}=-e_{j i}$. The cycle space $Z_{n+1}$ of $K_{n+1}$ is subspace of the edge space spanned by the length 3 cycles,

$$
e_{i j}+e_{j k}+e_{k i}, \quad(i, j, k \text { pairwise unequal }) .
$$


A basis is furnished by those 3 cycles visiting $n+1$, which we write as

$$
y_{i j}=e_{i j}+e_{j(n+1)}+e_{(n+1) i}, \quad(1 \leq i<j \leq n) .
$$

Notice that $y_{j i}=-y_{i j}$ and that the transpositions $(k(n+1))$ act by the rule:

$$
(k(n+1)) y_{i j}= \begin{cases}y_{i j}+y_{j k}+y_{k i} & k \notin\{i, j\}, \\ -y_{i j} & k \in\{i, j\} .\end{cases}
$$

The Gale dual of $\mathcal{A}_{n+1}$ is the arrangement in $Z_{n+1}$ given by the $\left(\begin{array}{c}n+1 \\ 2\end{array}\right)$ many hyperplanes,

$$
\begin{aligned}
y_{i j}, & (1 \leq i<j \leq n) \\
\sum_{j} y_{i j}, & (1 \leq i \leq n) .
\end{aligned}
$$

\subsection{The internal zonotopal algebra of the Gale dual of the braid arrangement}

We start with the following result.

Proposition 3.1 The Hilbert series of the quotient $\operatorname{Sym}\left(Z_{n+1}\right) / I_{\mathcal{A}_{n+1}^{\perp},-2}$ is given by

$$
(1+q)(1+2 q) \cdots(1+(n-1) q) \text {. }
$$

Proof: The Hilbert series is obtained by computing $q^{n} T_{\mathcal{A}_{n+1}}(1 / q, 0)$. This is obtained by massaging the Tutte polynomial evaluation $(-1)^{n} q T_{\mathcal{A}_{n+1}}(1-q, 0)=q(q-1) \cdots(q-n+1)$, which is the chromatic polynomial of $K_{n}$.

The dimensions of the graded pieces of the quotient are signless Stirling numbers of the first kind.

The following description of $I_{\mathcal{A}_{n+1}^{\perp},-2}$ is new, although the ideal appearing in it has arisen before.

Theorem 3.2 The ideal $I_{\mathcal{A}_{n+1}^{\perp},-2}$ is equal to

$$
\left\langle y_{i j}^{2}: 1 \leq i<j \leq n\right\rangle+\left\langle y_{i j} y_{k i}+y_{j i} y_{k j}+y_{k i} y_{j k}: 1 \leq i<j<k \leq n\right\rangle
$$

Let $J$ denote the ideal in question. We will prove the theorem using two lemmas, although there are many different ways to prove the result. The first lemma states that $J \subset I_{\mathcal{A}_{n+1}^{\perp},-2}$. The second lemma states that the displayed generators of $J$ form a Gröbner basis, and we will use this to see that the dimension of $\operatorname{Sym}\left(Z_{n+1}\right) / J$, as a vector space, is $n$ !. This will force the two ideals to be equal.

Lemma 3.3 There is a containment $J \subset I_{\mathcal{A}_{n+1}^{\perp},-2}$.

Proof: Consider the function $\rho_{\mathcal{A}_{n+1}^{\perp}}$ used to define $I_{\mathcal{A}_{n+1}^{\perp}, k}$. If $z$ is the sum of the edges in an oriented cycle of $K_{n+1}$ then $\rho_{\mathcal{A}_{n+1}^{\perp}}(z)$ is equal to the number of edges in $z$. It follows that $y_{i j}^{2} \in I_{\mathcal{A}_{n+1}^{\perp}}, k$. Consider the cycle that travels from vertex $i$ to vertex $j$ to vertex $k$ and back to $i$. Writing this in terms of the basis described above, it is

$$
y_{i j}+y_{j k}+y_{k i}
$$


Taking the square of this and subtracting off the squared monomials gives

$$
y_{i j} y_{k i}+y_{i j} y_{j k}+y_{k i} y_{j k}
$$

The desired inclusion follows.

Lemma 3.4 The set of polynomials,

$$
y_{i j}^{2}, \quad(1 \leq i<j \leq n), \quad-y_{i j} y_{i k}+y_{i j} y_{j k}-y_{i k} y_{j k}, \quad(1 \leq i<j<k \leq n) .
$$

forms a Gröbner basis under any term order where the leading terms are underlined above.

Proof: We use the fact that syzygies of polynomials with relatively prime leading terms need not be computed [Ei], Exercise 15.20]. By symmetry, the computation reduces to the case when $n=4$. This case is easily checked with a computer (e.g., using [M2]).

Proof of Theorem 3.2; The ideal of leading terms of $J$ is $\left\langle y_{i j}^{2}: 1 \leq i<j \leq n\right\rangle+\left\langle y_{i j} y_{j k}: 1 \leq i<j<\right.$ $k \leq n\rangle$. It follows that a basis for $\operatorname{Sym}\left(Z_{n+1}\right) / J$ consists of square-free monomials in the $y_{i j}$ that avoid $y_{i j} y_{i k}$, for $1 \leq i<j<k \leq n$. These monomials are in obvious bijection with the set of decreasing forests on $n$ vertices. These are forests with vertex set $[n]$ where, in every component, each path directed away from the largest vertex in that component decreases. Adding a vertex labeled $n+1$ and connecting the largest vertex in each component to $n+1$, we obtain a bijection between decreasing forests on $n$ vertices, and decreasing trees on $n+1$ vertices. By [EC1, Proposition 1.5.5], there are exactly $n$ ! such trees.

It follows that the dimension of $\operatorname{Sym}\left(Z_{n+1}\right) / J$ as a vector space is $n$ !. Since this is the dimension of $\operatorname{Sym}\left(Z_{n+1}\right) / I_{\mathcal{A}_{n+1}^{\perp},-1}$ and there is a containment between $J$ and $I_{\mathcal{A}_{n+1}^{\perp},-1}$, the two ideals are equal.

We recall the bijective proof of [EC1, Proposition 1.5.5]. Given a permutation $w=w_{1} \ldots w_{n} \in S_{n}$ we define a decreasing tree on vertex set $[n+1]$ as follows. Write $w^{\prime}=w_{1} \ldots w_{n}(n+1)$, and for $i \leq n$ declare the parent of $w_{i}^{\prime}$ to be first $w_{j}^{\prime}$ with $j>i$ and $w_{i}^{\prime}<w_{j}^{\prime}$.

With Theorem 3.2 in hand, one sees that $I_{\mathcal{A}_{n+1}^{\perp},-1}$ has been studied before by Mathieu [Ma, Theorem 3.1] and Gaiffi [Ga]. In the former work, $I_{\mathcal{A}_{n+1}^{\perp},-1}^{\perp}$ is connected to space of multilinear free Poisson polynomials in $n$ variables.

Remark 3.5 The action of the symmetric group $S_{n+1}$ on $\operatorname{Sym}\left(Z_{n+1}\right) / I_{\mathcal{A}_{n+1}^{\perp},-2}$ is obvious from our coordinate free description. In the coordinates $y_{i j}(1 \leq i<j \leq n)$ the extension of the natural action of $S_{n}$ on the quotient via equation [2] is an important result of both [Ma] and [Ga]. The action of $S_{n+1}$ on the Lie representation appears to have first been observed by Kontsevich.

Theorem 3.6 ([Ma, Theorem 4.4]) Let E denote the representation of $S_{n}$ obtained by grading the standard representation $\mathbf{C}^{n}$ in the following way: The trivial representation is in degree zero, while its unique $S_{n}$-invariant complement is in degree 1 . There is an isomorphism of $S_{n}$-representations,

$$
\operatorname{Res}_{S_{n}}^{S_{n+1}} \operatorname{Sym}\left(Z_{n+1}\right) / I_{\mathcal{A}_{n+1}^{\perp},-1} \approx \operatorname{Sym}\left(Z_{n}\right) / I_{\mathcal{A}_{n}^{\perp},-1} \otimes E
$$

As a representation of $S_{n}$, the character of $\operatorname{Sym}\left(Z_{n+1}\right) / I_{\mathcal{A}_{n+1}^{\perp},-1}$ is given by the sign twisted Lie character $\operatorname{sign} \otimes \operatorname{Ind}_{\langle(12 \cdots n)\rangle}^{S_{n}} e^{2 \pi i / n}$. As a representation of $S_{n-1}$, it is isomorphic to the regular representation. As a representation of $S_{n+1}, \operatorname{Sym}\left(Z_{n+1}\right) / I_{\mathcal{A}_{n+1}^{\perp},-1}$ is isomorphic to the sign twisted Whitehouse representation $[R W]$. 
For an introduction to the Whitehouse representation and its relation to the Lie representation, see Robinson and Whitehouse [RW], as well as the excellent slides of Stanley [St].

\section{The type B reflection arrangement}

We consider the arrangement $\mathcal{B}_{n}$ in $\mathbf{C}^{n}$ defined as the vanishing of the linear forms,

$$
x_{i}, \quad(1 \leq i \leq n), \quad x_{j}+x_{i}, \quad(1 \leq i<j \leq n), \quad x_{j}-x_{i}, \quad(1 \leq i<j \leq n) .
$$

This is the (complexified) arrangement of reflecting hyperplanes of the root system of type B and that of type $\mathrm{C}$, also known as the hyperoctohedral arrangement.

The Weyl group of the type $\mathrm{B} / \mathrm{C}$ root system in $\mathbf{C}^{n}$ is the hyperoctohedral group $B_{n}$. This is the group of signed $n$-by- $n$ permutation matrices. As such, elements of $B_{n}$ can be thought of as permutations $w$ of $\{ \pm 1, \pm 2, \ldots, \pm n\}$ satisfying $w(-i)=-w(i)$ for all $1 \leq i \leq n$. We will write elements of $B_{n}$ as "one-line permutations" and in disjoint cycle notation. We will usually write $\bar{i}$ instead of $-i$.

\subsection{The Gale dual of $\mathcal{B}_{n}$}

There is a natural candidate for the Gale dual of $\mathcal{B}_{n}$ that respects, in a suitable sense, the action of the hyperoctohedral group $B_{n}$ on complex $n$-space. The Gale dual of $\mathcal{B}_{n}$ will be a subspace of the space of linear functions defined on the $n^{2}$ hyperplanes in $\mathcal{B}_{n}$. This latter space comes with a distinguished basis $\left\{f_{i}: 1 \leq i \leq n\right\} \cup\left\{f_{i j}^{ \pm}: 1 \leq i<j \leq n\right\}$, in bijection with the hyperplanes in $\mathcal{B}_{n}$. Using this distinguished basis to define an orthonormal basis, we can identify subspaces of this space of functionals with their duals.

We adhere to the following conventions:

$$
f_{j i}^{+}=f_{i j}^{+}, \quad f_{j i}^{-}=-f_{i j}^{-} .
$$

There is a natural action of $B_{n}$ on the space of linear functions on the hyperplanes in $\mathcal{B}_{n}$. Permutations of the form $(i j)(\bar{i} \bar{j})$ act by permuting subscripts of the $f \mathrm{~s}$, while permutations of the form $(k \bar{k})$ act by the following rules:

$$
(k \bar{k}) f_{i}=\left\{\begin{array}{ll}
f_{i} & i \neq k, \\
-f_{i} & i=k .
\end{array} \quad(k \bar{k}) f_{i j}^{ \pm}= \begin{cases}f_{i j}^{ \pm} & k \notin\{i, j\} \\
f_{i j}^{\mp} & i=k \\
-f_{i j}^{\mp} & j=k .\end{cases}\right.
$$

Definition 4.1 Denote by $R_{n}$ the subspace offorms on $\mathcal{B}_{n}$ spanned by

$$
y_{i j}=f_{i j}^{-}-\left(f_{j}-f_{i}\right), \quad z_{i j}=f_{i j}^{+}-\left(f_{j}+f_{i}\right), \quad(1 \leq i<j \leq n) .
$$

This is the $n(n-1)$ dimensional vector space of linear relations among the linear forms on $\mathcal{B}_{n}$, and the displayed vectors form a basis for this space. $R_{n}$ is at once seen to have a $B_{n}$ action. We have the following consequences (among others) of the above rules:

$$
y_{j i}=-y_{i j}, \quad z_{j i}=z_{i j}, \quad(i \bar{i}) y_{i j}=z_{i j}, \quad(j \bar{j}) y_{i j}=-z_{i j} .
$$

The Gale dual $\mathcal{B}_{n}^{\perp}$ of $\mathcal{B}_{n}$ is the arrangement in $R_{n}$ defined by the vanishing of the $n^{2}$ linear forms

$$
y_{i j}, z_{i j} \quad(1 \leq i<j \leq n), \quad \sum_{j}\left(y_{i j}-z_{i j}\right) \quad(1 \leq i \leq n)
$$

Our first goal is to describe $I_{\mathcal{B}_{n}^{\perp},-2}$ in a form similar to that of Theorem 3.2 
Theorem 4.2 The ideal $I_{\mathcal{B}_{n}^{\perp},-2}$ is equal to

$$
\begin{aligned}
J=\left\langle y_{i j}^{2}, z_{i j}^{2}, y_{i j} z_{i j}: 1 \leq i<j \leq n\right\rangle+\left\langle y_{j i} y_{i k}+y_{i j} y_{j k}+y_{i k} y_{k j},\right. \\
\left.\quad z_{j i} y_{i k}-z_{i j} z_{j k}-y_{i k} z_{k j}: 1 \leq i<j<k \leq n\right\rangle .
\end{aligned}
$$

\subsection{The proof of Theorem 4.2}

We will need the following result.

Proposition 4.3 The quotient $\operatorname{Sym}\left(R_{n}\right) / I_{\mathcal{B}_{\bar{n}}^{\perp},-2}$ has Hilbert series

$$
(1+2 q)(1+4 q) \cdots(1+(2 n-2) q) .
$$

Proof: This follows in similar way to Proposition 3.1. First apply Theorem 2.1 to see that we must evaluate the Tutte polynomial of $\mathcal{B}_{n}$ at $(1 / q, 0)$. Then use a result of Zaslavsky [Za, Theorem 3] that relates the chromatic polynomial of $K_{n}$ with its signed version $\pm K_{n}$.

We will prove the theorem after first proving two lemmas.

Lemma 4.4 There is a containment of ideals $J \subset I_{\mathcal{B}_{n}^{\perp},-2}$.

Proof: The following linear forms define lines of the arrangement $\mathcal{B}_{n}^{\perp}$ :

$$
y_{i j}, \quad z_{i j}, \quad y_{i j} \pm z_{i j}, \quad y_{i j}+y_{j k}+y_{k i}, \quad z_{i j}-z_{j k}+y_{k i} .
$$

The number of hyperplanes that each of these does not lay on is 3 , hence each of their squares is in $I_{\mathcal{B}_{n}^{\perp},-2}$. Subtracting off the squared monomials from the squares of the latter three linear forms gives us the generators of $J$.

Lemma 4.5 The top degree component of $\operatorname{Sym}\left(R_{n}\right) / J$ is spanned by monomials of the form

$$
x_{w(1) w(2)} x_{w(2) w(3)} \ldots x_{w(n-1) n}
$$

where $x \in\{y, z\}$ and $w \in S_{n}$.

Proof: Recall that $J$ is the sum of two ideals:

$$
\begin{aligned}
& J_{1}=\left\langle y_{i j}^{2}, z_{i j}^{2}, y_{i j} z_{i j}: 1 \leq i<j \leq n\right\rangle, \\
& J_{2}=\left\langle y_{j i} y_{i k}+y_{i j} y_{j k}+y_{i k} y_{k j}, z_{j i} y_{i k}+z_{i j} z_{j k}-y_{i k} z_{k j}: 1 \leq i<j<k \leq n\right\rangle .
\end{aligned}
$$

Using just $J_{1}$, the non-zero monomials in the quotient are in obvious bijection with graphs on $[n]$, where the edges are signed with exactly one of $y$ or $z$. We claim that all these graphs are acyclic.

Say that a monomial corresponds to a graph with a length three cycle. Using the relations in $J_{2}$ we may rewrite the given monomial as a sum of elements in $J_{1}$. Hence, this monomial is zero in the quotient.

Suppose that we choose a monomial that corresponds to a graph $G$ with a cycle of length larger than three. Using, again, the relations in $J_{2}$ we find that the given monomial for $G$ can be written as a linear combination of monomials corresponding to two graph $G^{\prime}$ and $G^{\prime \prime}$, each with cycles of smaller length. See Figure 1 By induction, this monomial is zero in the quotient. 


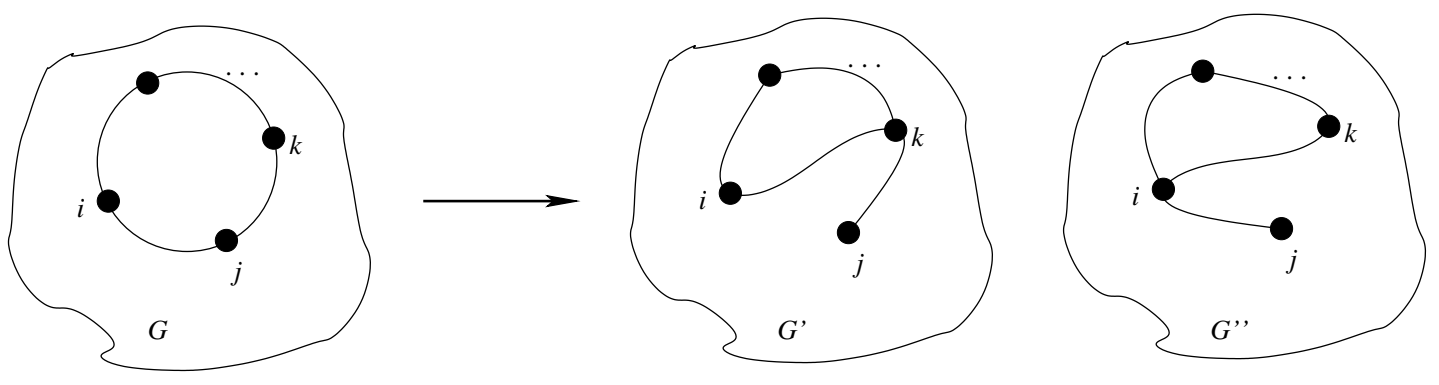

Fig. 1: Rewriting a monomial corresponding to a graph with a cycle of length larger than 3 in terms of two monomials whose graphs have cycles of smaller length.

We conclude that the non-zero monomials in the quotient correspond to acyclic graphs. It follows that the monomials of maximum degree correspond to trees, since the top degree non-zero component of $\operatorname{Sym}\left(R_{n}\right) / J$ is in degree at least $n-1$.

Given a monomial and corresponding tree $T$ with edges signed $y$ or $z$, assume that the degree of $n$ is larger than 1. Take the two largest indexed children of $n$. These come from a factor $x_{i n} x_{j n}$ in the monomial. Using $J_{2}$, we can rewrite this monomial as a sum of two monomials corresponding to graphs where $n$ has smaller degree than when we started. By an obvious induction, this monomial is in the span of the monomials of the form

$$
x_{w(1) w(2)} x_{w(2) w(3)} \ldots x_{w(n-1) n},
$$

where $x \in\{y, z\}$. This completes the proof of the lemma.

Proof of Theorem 4.2; Applying the same idea used in the proof of Lemma 4.5 we can identify a spanning set of monomials for $\operatorname{Sym}\left(R_{n}\right) / J$. A spanning set is given by monomials in bijective correspondence with forests on $[n]$, each component of which is a path with edges signed $y$ or $z$, and each path having its largest labeled vertex of degree one.

As computed in Lemma 4.5 the number of such forests which are connected is $2^{n-1}(n-1) !$. Using the exponential formula, the number of forests in question has exponential generating function

$$
\exp \left(\sum_{n=1}^{\infty} 2^{n-1}(n-1) ! x^{n} / n !\right)=\frac{1}{\sqrt{1-2 x}}=\sum_{n=0}^{\infty}(1 \cdot 3 \cdot 5 \cdots(2 n-1)) \frac{x^{n}}{n !} .
$$

Thus, we have found a spanning set of $1 \cdot 3 \cdot 5 \cdots(2 n-1)$ monomials of $\operatorname{Sym}\left(R_{n}\right) / J$. Since this is the dimension of $\operatorname{Sym}\left(R_{n}\right) / I_{\mathcal{B}_{n},-2}$ and we have a containment $J \subset I_{\mathcal{B}_{n},-2}$, the containment must be an equality.

As an immediate corollary of the theorem we obtain the main result of our work:

Theorem 4.6 As a representation of the Weyl group $B_{n-1}$, the top degree component of $\operatorname{Sym}\left(R_{n}\right) / I_{\mathcal{B}_{n}^{\perp},-2}$ carries the regular representation.

Proof: It has the correct dimension, $2^{n-1}(n-1)$ !, and was just shown to be spanned by the $B_{n-1}$ orbit of $y_{12} y_{23} \cdots y_{(n-1) n}$. The result follows. 


\subsection{Recurrence}

In this section we give an analog of the first part of Theorem 3.6. For this we must recall that that the irreducible representations of $B_{n}$ are in bijective correspondence with pairs of partitions $(\lambda, \mu)$, where $|\lambda|+|\mu|=n$ (see [Ma, App. B]). We denote the irreducible representation indexed by $(\lambda, \mu)$ by $S^{(\lambda, \mu)}$.

Theorem 4.7 Let $E$ be the graded representation of $B_{n-1}$ that is trivial in degree zero and is the direct sum of $S^{((n-2,1), \emptyset)}$ and $S^{((1),(n-2))}$ in degree one. There is an isomorphism of $B_{n-1}$ representations

$$
\operatorname{Res}_{B_{n-1}}^{B_{n}} \operatorname{Sym}\left(R_{n}\right) / I_{\mathcal{B}_{n}^{\perp},-2} \approx \operatorname{Sym}\left(R_{n-1}\right) / I_{\mathcal{B}_{n-1}^{\perp},-2} \otimes E .
$$

Proof (sketch): The idea is to separate out the monomials of the form $y_{i j}$ and $z_{i j}$ with $1 \leq i<j<n$, from those of the form $y_{i n}$ and $z_{i n}$ with $1 \leq i<n$. Both of these collections span a $B_{n-1}$ stable subspace of $R_{n}$, and the character of the latter representation is isomorphic to the degree one component of $E$.

\section{Decreasing trees of type B/C}

In this section we introduce a combinatorial curiosity, which we believe to be the proper analog of decreasing trees to the hyperoctohedral setting.

Definition $5.1 A \pm$ tree on $n$ vertices is a rooted tree with vertex set $[n]$ and root vertex $n$, together with a $\{+,-\}$-coloring of its edges. A decreasing \pm tree on $n$ vertices is a \pm tree on $n$ vertices such that on any path directed away from the root,

1. along every edge labeled - the vertex labels decrease,

2. for $i<j$ and arbitrary $k$, there is no path of the form

$$
j \stackrel{-}{\longrightarrow} i \stackrel{+}{\longrightarrow} k,
$$

3. for all $i_{1}<i_{2}<i_{3}<i_{4}$, there is no subpath of any of the three forms, or their reverse,

$$
\begin{aligned}
& i_{4} \stackrel{+}{\longrightarrow} i_{1} \stackrel{+}{\longrightarrow} i_{2} \stackrel{+}{\longrightarrow} i_{3}, \\
& i_{4} \stackrel{+}{\longrightarrow} i_{1} \stackrel{+}{\longrightarrow} i_{3} \stackrel{+}{\longrightarrow} i_{2}, \\
& i_{4} \stackrel{+}{\longrightarrow} i_{2} \stackrel{+}{\longrightarrow} i_{1} \stackrel{+}{\longrightarrow} i_{3} .
\end{aligned}
$$

If we ignore the possibility of edges labeled + this definition reduces to that of decreasing trees given previously.

Example 5.2 Here are the 8 decreasing \pm trees on 3 vertices.
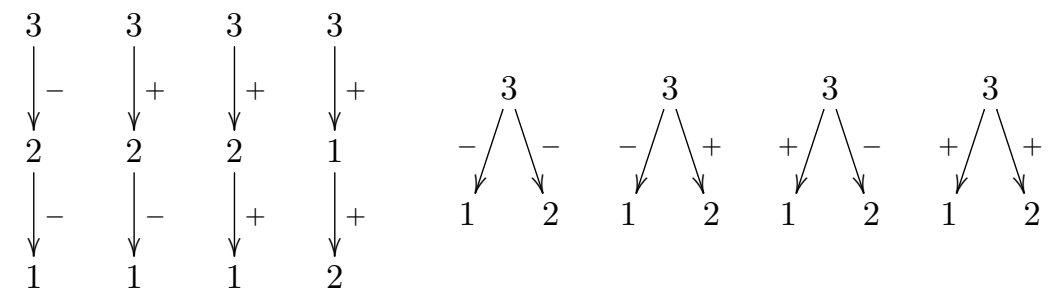
Example 5.3 There are 48 decreasing \pm trees on 4 vertices. Exactly 8 such trees are isomorphic to a path rooted at an endpoint, and these are displayed below.

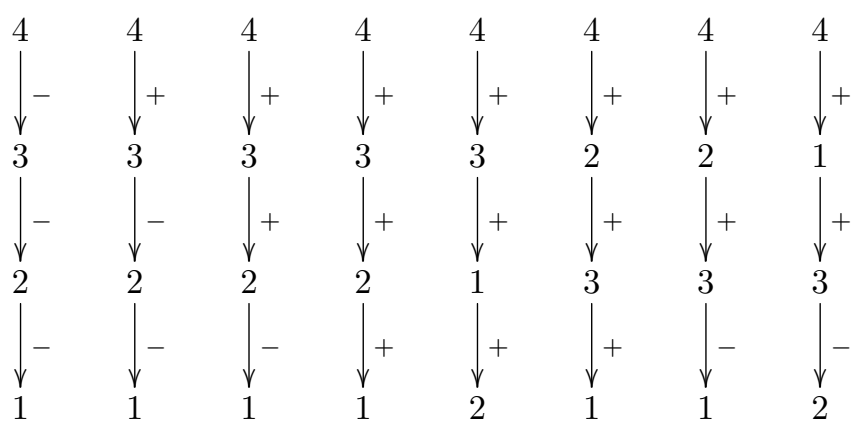

Theorem 5.4 The number of decreasing \pm trees on $n$ vertices is $2^{n-1}(n-1) !$.

Proof: We know from the proof of Lemma 4.5 that the graphs corresponding to monomials not in $I_{\mathcal{B}_{n}^{\perp},-2}$ are \pm trees. We now show that the monomials of decreasing \pm trees form a basis for the quotient $\operatorname{Sym}\left(R_{n}\right) / I_{\mathcal{B}_{n}^{\perp},-2}$.

For this, we claim that a Gröbner basis of $I_{\mathcal{B}_{\frac{1}{n},-2}}$ is furnished by the following monomials:

$$
y_{i j}^{2}, \quad z_{i j}^{2}, \quad y_{i j} z_{i j}, \quad z_{i j} z_{j k} z_{k i}
$$

where $i, j$ and $k$ range of distinct triples of integers, as well as the polynomials

$$
\underline{y_{j i} y_{i k}}+y_{i j} y_{j k}+y_{i k} y_{k j}, \quad \underline{z_{j i} y_{i k}}+z_{i j} z_{j k}-y_{i k} z_{k j},
$$

where the indices range over distinct tuples of integers, and finally given $1 \leq i_{1}<i_{2}<i_{3}<i_{4} \leq n$ the polynomials,

$$
\begin{aligned}
& z_{i_{1} i_{2}} z_{i_{1} i_{3}} z_{i_{2} i_{4}}-z_{i_{1} i_{2}} z_{i_{1} i_{3}} z_{i_{3} i_{4}}-z_{i_{1} i_{2}} z_{i_{2} i_{4}} z_{i_{3} i_{4}}+\underline{z_{i_{1} i_{3}} z_{i_{2} i_{4}} z_{i_{3} i_{4}}}, \\
& z_{i_{1} i_{3}} z_{i_{2} i_{3}} z_{i_{1} i_{4}}-z_{i_{1} i_{3}} z_{i_{2} i_{3}} z_{i_{2} i_{4}}-z_{i_{1} i_{3}} z_{i_{1} i_{4}} z_{i_{2} i_{4}}+\underline{z_{i_{2} i_{3}} z_{i_{1} i_{4}} z_{i_{2} i_{4}}}, \\
& z_{i_{1} i_{2}} z_{i_{2} i_{3} i_{3}} z_{i_{1} i_{4}}-z_{i_{1} i_{2}} z_{i_{2} i_{3}} z_{i_{3} i_{4}}-z_{i_{1} i_{2}} z_{i_{1} i_{4}} z_{i_{3} i_{4}}+\underline{z_{i_{2} i_{3}} z_{i_{1} i_{4}} z_{i_{3} i_{4}}}
\end{aligned}
$$

We use any term order where the underlined monomials are leading terms (such as graded-reverse lexicographic order with the $y$ 's coming before the $z$ 's).

To see this one applies the Buchberger algorithm to the generators of $I_{\mathcal{B}_{n}^{\perp},-2}$ given in Theorem 4.2 As before, we use [Ei Exercise 15.20] to reduce the computation to the case $n=4$, since we need not resolve the syzygies of pair of polynomials with relatively prime leading terms. This case is, once again, handled at once by a computer to produce the described Gröbner basis.

A bijective proof of this result generalizing the one for ordinary decreasing trees is not known.

\section{Acknowledgments}

Special thanks to Steve Klee for useful discussions on \pm decreasing trees and to Brendon Rhoades for early discussions on the material in Section 3 . 


\section{References}

[AP10] F. Ardila and A. Postnikov. Combinatorics and geometry of power ideals. Trans. Amer. Math. Soc. 362 (2010), no. 8, 4357-4384.

[AP15] F. Ardila and A. Postnikov. Correction to "Combinatorics and geometry of power ideals": two counterexamples for power ideals of hyperplane arrangements. Trans. Amer. Math. Soc. 367 (2015), no. 5, 3759-3762.

[Be] N. Bergeron. A hyperoctahedral analogue of the free Lie algebra. J. Combin. Theory Ser. A 58 (1991), no. 2, 256-278.

[BR] A. Berget and B. Rhoades. Extending the parking space. J. Combin. Theory Ser. A no. 123 (2014), 43-56.

[Do] M. Douglas. On the cohomology of an arrangement of type $B_{l}$. J. Algebra 147 (1992), no. 2, 265282.

[Ei] D. Eisenbud. Commutative algebra. With a view toward algebraic geometry. Graduate Texts in Mathematics, 150. Springer-Verlag, New York, 1995.

[Ga] G. Gaiffi. The actions of $S_{n}$ and $S_{n+1}$ on the cohomology ring of the coxeter arrangement of type $A_{n+1}$. Manuscripta Math. 91 (1996), no. 1, 83-94.

[HR] O. Holtz and A. Ron. Zonotopal algebra. Adv. Math. 227 (2011), no. 2, 847-894.

[LS] G. Lehrer and L. Solomon. On the action of the symmetric group on the cohomology of the complement of its reflecting hyperplanes. J. Algebra 104 (1986), no. 2, 410-424.

[Ma] I.G. Macdonald. Symmetric functions and Hall polynomials (Second edition). Oxford Mathematical Monographs, Oxford University Press. New York, 1995.

[M2] D. Grayson and M. Stillman. Macaulay2, a software system for research in algebraic geometry. Available at http://www.math.uiuc.edu/Macaulay2/(retrieved Nov. 2015).

[Ma] O. Mathieu. Hidden $\Sigma_{n+1}$-actions. Comm. Math. Phys. 176 (1996), no. 2, 467-474.

[OS] P. Orlik and L. Solomon. Combinatorics and topology of complements of hyperplanes. Invent. Math. 56 (1980), no. 2, 167-189.

[EC1] R. Stanley. Enumerative combinatorics. Volume 1. Second edition. Cambridge Studies in Advanced Mathematics, 49. Cambridge University Press, Cambridge, 2012.

[RW] A. Robinson and S. Whitehouse. The tree representation of $\Sigma_{n+1}$. J. Pure Appl. Algebra 111 (1996), no. 1-3, 245-253.

[St] R. Stanley. The Whitehouse Module. Available at http://www-math.mit.edu/ rstan/ transparencies/whouse.pdf (retrieved Nov. 2015).

[Za] T. Zaslavsky. The geometry of root systems and signed graphs. Amer. Math. Monthly 88 (1981), no. 2,88105 . 